\title{
Qualität in der Medizin: Was sich kaum messen lässt*
}

\section{Hans Stalder}

Prof. Dr. med., Mitglied der Redaktion

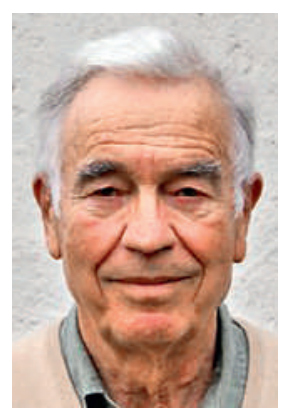

Wenn unser Vorgehen einer bestimmten Linearität folgt, wenn beispielsweise die Anamnese und die klinische Untersuchung zur Differentialdiagnose und nach ergänzenden Untersuchungen zur Diagnose und zur Behandlung führen, sind Qualitätsmassnahmen theoretisch möglich, u.a. auch durch das Einhalten von Guidelines, selbst wenn das manchmal problematisch ist $[1,2]$. Letztlich zählt jedoch - ganz unabhängig vom Qualitätsansatz - nicht das Verfahren, sondern das Ergebnis, und in der Medizin ist das Ziel, die Lebensqualität der Patienten zu verbessern.

Mit der Definition der QALYs (quality-adjusted life years), einer Kennzahl zur Bewertung des Nutzens eines medizinischen Eingriffs - beispielsweise einer neuen Behandlung in der Onkologie -, wurde versucht, Lebensqualität zu messen. Fragebögen wie der EQ-5D ermitteln Parameter wie Mobilität, Schmerzen, Selbstversorgung, Angst, alltägliche Aktivitäten [3]. Für jedes dieser Kriterien werden Punkte vergeben und in der Folge addiert. Daraus ergibt sich ein Messwert für die Lebensqualität. Bald wurde klar, dass die Ergebnisse dieser Fragebögen nicht immer dem entsprechen, was der Patient empfindet. Deshalb wurde eine etwas allgemeinere Frage hinzugefügt, wie beispielsweise «Wie ist Ihr Gesundheitszustand heute?» [3]. Ihr Gesundheitszustand? Sicherlich nicht nach der Definition der WHO - «ein Zustand des voll-

\section{Bald wurde klar, dass die Ergebnisse dieser Fragebögen nicht immer dem entsprechen, was der Patient empfindet.}

ständigen körperlichen und geistigen Wohlergehens und nicht nur das Fehlen von Krankheit oder Gebrechen"-, denn diese passt ja nie auf einen unserer Patienten. Passen würde da schon eher der Gesundheitbegriff nach einer neuen Definition einer Expertengruppe: "Gesundheit ist die Fähigkeit, sich anzupassen und selbst zu managen» [4].

Die Fähigkeit, sich anzupassen und selbst zu managen, ist eine der Besonderheiten komplexer Systeme, die alle Lebewesen auszeichnet - und damit auch unsere Patienten. Zu den weiteren Besonderheiten dieser Systeme zählen die Unvorhersehbarkeit - kleine Interventionen können unvorhersehbare Wirkungen zeitigen und die Sensibilität für den ursprünglichen Zustand, d.h. für die Vergangenheit, was bedeutet, dass zwei Patienten sich nie vollkommen gleichen, selbst wenn sie dieselbe Pathologie vorweisen. Zudem lässt sich ein komplexes System nicht in seine Einzelteile zerlegen, ohne dass es seine Identität verliert. Man kann es deshalb auch nicht einfach mittels eines Fragebogens analysieren und glauben, es dadurch in seiner Gesamtheit zu verstehen. Schliesslich können komplexe Systeme mit anderen in Kontakt treten. Und eines dieser anderen komplexen Systeme mit all seinen Besonderheiten, mit denen der Patient in Kontakt tritt, ist der Arzt.

\section{Der Kontakt zwischen Arzt und Patient ist in} doppelter Weise einzigartig: eine Begegnung zweier komplexer Systeme.

Der Kontakt zwischen Arzt und Patient ist somit in doppelter Weise einzigartig: eine Begegnung zweier komplexer Systeme. Jeder Beteiligte hat seine eigene Vergangenheit und seine eigenen individuellen Erfahrungen. Die Qualität dieses Kontaktes und vor allem die Qualität des Ergebnisses dieses Kontaktes, nämlich die Verbesserung der Lebensqualität des Patienten, lässt sich bestenfalls erahnen, aber niemals genau messen, auch nicht mit den ausgefeiltesten Fragebögen. Und sie lässt sich vor allem nicht in wirtschaftliche Begriffe übersetzen ...

\section{Literatur}

1 Stalder H. Qualität in der Medizin: Was (potentiell) messbar ist. Schweiz Ärztezeitung. 2015;96(43):1588.

2 Woolf SH, Grol R, Hutchinson A, Eccles M, Grimshaw J. Clinical guidelines: potential benefits, limitations, and harms of clinical guidelines. BMJ. 1999;318:527-30.

3 http://phi.uhce.ox.ac.uk/inst_types.php

4 Huber M, Knottnerus JA, Green L, van der Horst H, Jadad AR, Kromhout D, et al. How should we define health? BMJ. 2011;343:235-7. 